\title{
THE EVOLUTIONARY STATUS OF WNL STARS
}

\author{
PAUL A. CROWTHER ${ }^{1}$, LINDA J. SMITH ${ }^{1}$ and D. JOHN HILLIER ${ }^{2}$ \\ ${ }^{1}$ Department of Physics and Astronomy, University College London, \\ Gower Street, London, WC1E 6BT U.K. \\ ${ }^{2}$ Department of Physics and Astronomy, University of Pittsburgh, \\ 3941 O'Hara Street, Pittsburgh, PA 15260, U.S.A.
}

\begin{abstract}
We discuss the evolutionary and mass loss implications of recent non-LTE analyses of late WN (WNL) stars in the Galaxy, LMC and M33 using the Wolf-Rayet standard model and address the observed dichotomy of WNL stars discussed by Moffat (1989). Individual subtypes belong to two distinct groups. We find that the single WNL+abs and WN7 stars evolve directly from very massive $O$ stars $\left(60-100 \mathrm{M}_{\odot}\right)$ with the former intimately related to extreme Of stars and found exclusively in the youngest clusters in our Galaxy. Conversely, the observational properties and chemistries of WN8-10 stars suggest that they are descended from lower initial mass progenitors $\left(\sim 25-60 \mathrm{M}_{\odot}\right)$. These stars are either dormant LBVs $(=\mathrm{WN} 9-10)$ or at a phase immediately after this stage $(=\mathrm{WN} 8)$ although a previous RSG phase cannot be excluded. Included in this subgroup are the LMC and M33 Ofpe/WN9 stars, re-classified as WN9-10 since they appear to be genuine WN stars.
\end{abstract}

Key words: stars: Wolf-Rayet - atmospheres - abund ances - winds - mass-loss

\section{Introduction}

All single stars with initial masses greater than around $30-40 \mathrm{M}_{\odot}$ are believed to evolve to the WR phase. Of these, the late WN stars (WNL) are the most luminous and the least chemically evolved, indicating that the most massive stars enter the WR phase as WNL stars, although the exact sequence leading to this stage is not well established. The commonly held view is that only very massive stars $\left(>120 \mathrm{M}_{\odot}\right)$ proceed directly to the WNL phase, while lower mass stars first pass through an intermediate stage of either a Luminous Blue Variable (LBV) for stars of initial mass $60-120 \mathrm{M}_{\odot}$ or a red supergiant (RSG) otherwise. Amongst the WNL stars, the earliest subtypes (WN6-7) have a high binary frequency, are located in clusters and show low intrinsic photometric variability. This is in marked contrast to the low binary frequency, dispersed spatial distribution, and high variability of later (WN8-9) subtypes (Moffat 1989).

In recent papers we have investigated the physical and chemical parameters of a sample of LBVs (Smith et al. 1994), LMC Ofpe/WN9 stars (Crowther et al. 1994a), Galactic WNL stars (Crowther et al. 1994a, b) and an M33 Ofpe/WN9 star (Crowther et al. these proc.). Tailored analyses using the non-LTE WR standard model of Hillier (1990) and high quality UV, optical and IR spectroscopy allow the determination of stellar parameters and chemical abundances and also the identification of inadequacies of the present model (see Hillier, these proceedings). 
From our sample, the Ofpe/WN9 and WNL+abs stars have apparently composite Of-WN spectral characteristics (Walborn 1982; Conti \& Bohannan 1989). We find that the Ofpe/WN9 stars, located in the LMC and M33, with apparently Of photospheric lines, are in fact spectrally very late WN stars (WN9-10) since these features are weak P Cygni profiles (Crowther $e t$ al. 1994a; these proc.). This re-classification avoids the incorrect assumption that Ofpe/WN9 stars represent an intermediate evolutionary stage between Ofpe and WN9 subtypes. Since these stars are also highly chemically evolved they should be included in the WR population of galaxies when comparing the number of $\mathrm{O}$ to $\mathrm{WR}$ stars with the predictions of evolutionary models. Similarly, the WNL+abs stars, while generally single, have absorption lines in their upper Balmer-Pickering series. Again, these lines are weak P Cygni wind profiles, implying that WNL classifications are appropriate.

\section{Physical parameters and evolutionary status}

We present the stellar parameters and chemistries of selected WNL stars in Table I and compare these with LBVs and $\mathrm{O}$ supergiants taken from the recent literature. We find that the properties of the WNL+abs stars are very similar to Of supergiants, suggesting a direct evolutionary connection. This confirms the suspicions of Walborn (1973) and Moffat (1978) that these stars represent a relatively early post-main sequence (MS) evolutionary stage of extremely massive stars and explains the ambiguous Of-WN spectral classification of such stars. WNL+abs stars appear to be exclusive to the youngest Galactic clusters (e.g., $\operatorname{Tr} 16$ ), have estimated masses in the range 35-55 $\mathrm{M}_{\odot}$, and progenitor masses of $60-100 \mathrm{M}_{\odot}$ (see also L.F. Smith et al. 1994). We suggest a direct evolutionary sequence from WN7+abs to WN7 stars (e.g., WR22 and WR78) from their spatial similarities and the continuity in morphology and physical parameters.

Turning to WN8-10 stars, there are strong observational similarities with LBVs. For instance, both populations are very rarely found in clusters or associations, they are variable, often show evidence for non-spherical winds, are rarely found in binaries and some have ejecta nebulae. Due to their advanced evolutionary status, many Galactic WN8 stars may be post-LBVs, although a post-RSG status cannot be excluded. Table I shows that the luminosity and chemistry of R84 (WN9) is identical to R71 (LBV). Since R84 is photometrically variable at a similar level to dormant LBVs (Stahl et al. 1984; Lamers 1987) it is probably a dormant LBV, or at a stage immediately after this phase. Recall that another Ofpe/WN9 (=WN9-10?) star, R127, quite unexpectedly, became an LBV in the early 1980's (Stahl et al. 1983). The past evolutionary histories of the remaining WN9-10 stars are less clear and although some fall below the Humphreys-Davidson limit (see Crowther et al. these proc.), the strong spectral and chemical similarities among our 
TABLE I

Comparison of WNL stars in the Galaxy (MW), Large Magellanic Cloud (LMC) and M33.

\begin{tabular}{|c|c|c|c|c|c|c|c|c|c|c|}
\hline Star & Sp.T & $\log T_{\text {eff }}$ & $\log L$ & $\log \dot{M}$ & $v_{\infty}$ & $\mathrm{H} / \mathrm{He}$ & $\mathrm{C} / \mathrm{N}$ & Status & Galaxy & Ref \\
\hline$\zeta$ Pup & O4I(n)f & 4.62 & 6.0 & -5.4 & 2200 & 5.0 & 0.12 & pre-WNL+abs? & MW & d \\
\hline WR25 & WN7+abs & 4.49 & 6.0 & -4.4 & 2480 & 4.5 & 0.08 & post-Of & MW & c \\
\hline WR22 & WN7+abs & 4.49 & 5.8 & -4.3 & 1785 & 3.2 & 0.08 & post-Of & MW & c \\
\hline HD152408 & O8:Iafpe & 4.52 & 6.0 & -4.7 & 960 & $\ldots$ & $\ldots$ & pre-WNL+abs? & MW & d \\
\hline WR108 & WN9+abs & 4.46 & 5.9 & -4.3 & 1170 & 1.5 & 0.10 & post-Of & MW & b \\
\hline WR78 & WN7 & 4.48 & 5.8 & -4.1 & 1385 & 0.5 & 0.02 & post-Of & MW & c \\
\hline Sk- $66^{\circ} 40$ & WN10 & 4.32 & 5.5 & -4.5 & 300 & 3.5 & 0.30 & dormant LBV? & LMC & b \\
\hline MCA1b & WN9-10 & 4.28 & 5.9 & -4.0 & 420 & 2.6 & 0.13 & dormant LBV? & M33 & e \\
\hline AG Car & WN11 & 4.32 & 6.0 & -4.2 & 250 & 2.4 & $\ldots$ & LBV & MW & $\mathbf{a}$ \\
\hline R84 & WN9 & 4.40 & 5.8 & -4.4 & 400 & 2.4 & 0.03 & dormant LBV? & LMC & b \\
\hline R71 & $\mathrm{B} 2.5 \mathrm{Ia}$ & 4.23 & 5.8 & -6.2 & 170 & 2.3 & 0.03 & LBV & LMC & b \\
\hline HD269927c & WN9 & 4.45 & 5.7 & -4.5 & 500 & 1.7 & 0.08 & post-LBV? & LMC & b \\
\hline WR40 & WN8 & 4.40 & 5.5 & -4.0 & 840 & 0.8 & 0.01 & post-LBV? & MW & c \\
\hline WR123 & WN8 & 4.41 & 5.5 & -4.0 & 970 & 0.1 & 0.02 & post-LBV? & MW & c \\
\hline
\end{tabular}

Notes. a: Smith et al. (1994); b: Crowther et al. (1994a) and references therein; c: Crowther et al. (1994b), d: Crowther et al. (1994c) and references therein; e: Crowther et al. (these proceedings)

sample of stars suggest that they too are closely related to LBVs. Initial masses of the WN8-10 stars are estimated to lie in the range $25-60 \mathrm{M}_{\odot}$.

In summary, we suggest the following evolutionary sequence for the most massive stars $\left(\mathrm{M}_{\text {initial }}>60 \mathrm{M}_{\odot}\right)$, $O \Rightarrow O f \Rightarrow W N L+a b s \Rightarrow W N \gamma$ ( $\Rightarrow W N E \Rightarrow W C \Rightarrow S N ?$ ?).

While WNL+abs stars are exclusive to our Galaxy, corresponding objects in the LMC may be the OIf/WN6-7 stars (Walborn 1986) which are also found in extremely young clusters. For lower initial mass $\left(25 \mathrm{M}_{\odot}<\mathrm{M}_{\text {initial }}<60 \mathrm{M}_{\odot}\right)$ stars, either the RSG or LBV $\left(\mathrm{M}_{\text {initial }} \geq 40 \mathrm{M}_{\odot}\right)$ phase is entered, $O \Rightarrow O f \Rightarrow R S G$ or $L B V \Leftrightarrow W N 9-10 \Rightarrow W N 8$ ( $\Rightarrow W N E \Rightarrow W C \Rightarrow S N$ ? ).

Our results support previous evidence for the 'luminosity discrepancy' between the theoretical and observed HRD location of the WR stars (Hamann 1994) in that observed luminosities are lower than those predicted. Recently, two different theoretical approaches have attempted to solve this problem. Meynet et al. (1994) have calculated evolutionary tracks with enhanced mass loss from the MS to the WNL phase, while Langer et al. (1994) have developed an alternative model with increased mass loss after the MS due to pulsational instabilities. Our findings show some similarities with these new evolutionary computations in that a hydrogen-rich WNL phase (= WNL+abs?) is predicted to evolve directly from very massive $\mathrm{O}$ stars. How- 

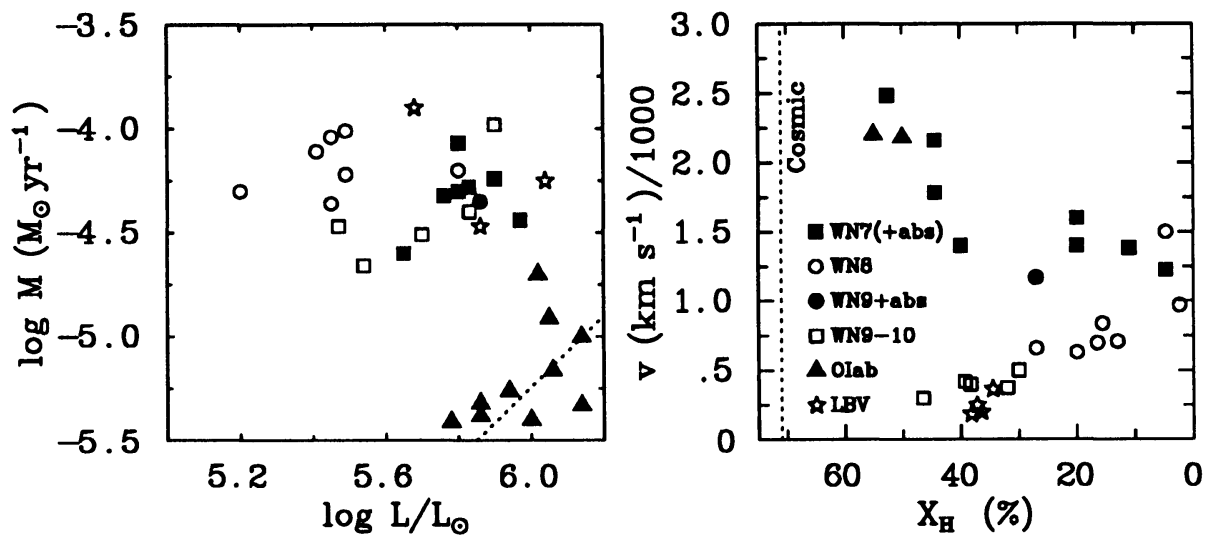

Fig. 1. Left panel: Mass-loss rates of LBVs, WNL stars and $O$ supergiants versus their luminosities. The $\dot{M}$-L relation of OB stars (Howarth \& Prinja 1989) is also shown (dashed line). Right panel: Terminal velocity versus hydrogen abundance (mass fraction) for WNL stars. Two distinct evolutionary sequences can clearly be identified.

ever, the mass loss rates used by Meynet et al. (1994) are inconsistent with the observed rates for O stars (e.g., Lamers \& Leitherer 1993) while Langer et al. (1994) predict an LBV phase between hydrogen-rich and hydrogenpoor WNL phases. In conflict with this scheme is the 'hydrogen-poor' WNL star WR108 (WN9+abs) which is morphologically a very extreme Of star (Walborn 1982) and is without an ejecta nebula, implying that evolution can proceed directly from an Of star to a 'hydrogen-poor' WNL star.

\section{Mass loss and variability}

The mass-loss rates of the WNL stars as a whole appear to be fairly independent of luminosity (see Fig. 1, left panel) in contrast to the well known $\dot{M}-\mathrm{L}$ relation for $\mathrm{OB}$ stars. We find that the WN8 stars have the most extreme $\dot{M} / \mathrm{L}$ ratios while some WNL+abs stars (e.g., WR25, WN7+abs) have values as little as a factor of two greater than some extreme $\mathrm{O}$ supergiants (e.g., HD 152408, O8:Iafpe), confirming the continuity in mass loss properties from Of to WNL stars identified by Lamers \& Leitherer (1993).

The most luminous WR stars are commonly believed to be the most variable (Moffat 1989). However, we find that those WNL stars with the fastest winds (i.e. WNL+abs and WN7 stars) are extremely stable, while those with low terminal velocities (WN8-10 stars) are highly variable (see also Robert et al. 1989). Figure 1 (right panel) clearly shows the evolutionary continuation from Of stars to the lower terminal velocity WNL+abs and WN7 stars. A sequence of increasing wind velocity from the WN9-10 and LBVs to the WN8 stars can also be identified (see also Table I).

One remaining question is that of the driving mechanism responsible for 
the extremely dense winds of the WNL stars. Prinja \& Smith (1992) have detected migrating opacity enhancements in WR24 (WN7+abs), analogous to those seen in $\mathrm{OB}$ winds, suggesting a common mechanism. Meanwhile, the theoretical predictions of pulsational instabilities investigated by Langer $e t$ al. (1994) are promising for WN8-10 stars and LBVs since rapid pulsations were first observed in one such star (WR40, WN8) by Blecha et al. (1992).

\section{References}

Blecha, A., Schaller, G., Maeder, A. 1992, Nature 360, 320

Conti, P.S., Bohannan, B. 1989, in: Davidson, K., Moffat, A.F.J. \& Lamers, H.J.G.L.M (eds.), Physics of Luminous Blue Variables, Proc. IAU Coll. No. 113 (Dordrecht: Kluwer), p. 297

Crowther, P.A., Hillier, D.J., Smith, L.J. 1994a, $A \& A$ in press

Crowther, P.A., Hillier, D.J., Smith L.J. 1994b, $A \& A$ in press

Crowther, P.A., Smith, L.J., Hillier, D.J., Schmutz, W 1994c, $A \& A$ in press

Hamann, W.-R. 1994, Sp. Sci. Rev. 66, 237

Hillier, D.J. 1990, $A \mathscr{E} A$ 231, 116

Howarth, I.D., Prinja, R.K. 1989, ApJ Suppl. 69, 527

Lamers, H.J.G.L.M. 1987, in: H.J.G.L.M. Lamers \& C.W.H. de Loore (eds.), Instabilities in Luminous Early-Type Stars (Dordrecht: Reidel), p. 99

Lamers, H.J.G.L.M., Leitherer, C. 1993, $A p J$ 412, 771

Langer, N., Hamann, W.-R., Lennon, M., Najarro, F., Pauldrach, A.W.A., Puls, J. 1994, $A \mathscr{E} A$ in press

Meynet, G., Maeder, A, Schaller, G., Schaerer, D., Charbonnel, C. 1994, A\&BA Suppl. 103, 97

Moffat, A.F.J. 1978, $A \& A 68,41$

Moffat, A.F.J. 1989, $A p J$ 347, 373

Prinja, R.K., Smith, L.J. 1992, $A \& A$ 266, 377

Robert, C., Moffat, A.F.J, Bastien, P., Drissen, L., St-Louis, N. 1989, ApJ 347, 1034

Smith, L.F., Meynet, G., Mermilliod, J-C. 1994, $A \mathcal{E} A$ in press

Smith, L.J., Crowther, P.A., Prinja, R.K. 1994, $A \cup A$ 281, 833

Stahl, O., Wolf, B., Klare, G., Cassatella, A., Krautter, J., Persi, P., Ferrari-Toniolo, M. 1983, $A \& A 127,49$

Stahl, O., Wolf, B., Leitherer, C., Zickgraf, F.J., Krautter, J., de Groot, M. 1984, $A \mathscr{E} A$ 140,459

Stahl, O. 1986, $A \& A 164,321$

Walborn, N.R. 1973, $A J$ 78, 1067

Walborn, N.R. 1982, ApJ 256, 452

Walborn, N.R. 1986, in: C.W.H. De Loore, A.J. Willis \& P. Laskarides (eds.), Luminous Stars and Associations in Galaxies, Proc. IAU Symp. No. 116 (Dordrecht: Reidel), p. 185

\section{DISCUSSION:}

Williams: Why is there a difference in cluster membership between the WN7 and WN8-11 stars?

Crowther: WN7 stars apparently descend directly from very massive progenitors found only in clusters with peculiar IMFs as evidenced from their concentration of very massive 03-6 stars (e.g. Tr16, HM1). In contrast WN8-11 stars result from lower mass progenitors which are not restricted to such clusters. While cluster member WN8 stars do exist (e.g. WR66, 105) their characteristics are more representative of WN7 stars (e.g. non-variable, high terminal velocity). 